\title{
CAPITUlation, AMBIGUOUS CLASSES AND THE COHOMOLOGY OF THE UNITS.
}

\author{
Cristian D. GonzÁlez-AviléS
}

\begin{abstract}
This paper presents results on both the kernel and cokernel of the $S$ capitulation map $C_{F, S} \rightarrow C_{K, S}^{G}$ for arbitrary finite Galois extensions $K / F$ of global fields (with Galois group $G$ ) and arbitrary finite sets of primes $S$ of $F$ (assumed to contain the archimedean primes in the number field case).
\end{abstract}

\section{Introduction.}

Let $K / F$ be a finite Galois extension of number fields with Galois group $G$, and let $C_{F}$ and $C_{K}$ denote, respectively, the ideal class groups of $F$ and $K$. The extension of ideals from $F$ to $K$ induces a natural capitulation map $j_{K / F}: C_{F} \rightarrow C_{K}^{G}$. An important problem in Number Theory is to determine the kernel of $j_{K / F}$, which is usually called the "capitulation kernel". The classical Principal Ideal Theorem of global class field theory (see [11, Theorem II.5.8.3, p.168] for a generalized version of this theorem) asserts that Ker $j_{K / F}$ is all of $C_{F}$ if $K$ is the Hilbert class field of $F$. This fact motivated, quite early on, a great deal of interest in the study of Ker $j_{K / F}$ for subfields $K$ of the Hilbert class field of $F$. As a result, most of the existing literature on the Capitulation Problem is concerned with the study of Ker $j_{K / F}$ for unramified abelian extensions $K$ of $F$ (see, e.g., [4, 12, 17, 25]) or, more generally, with the kernel (and cokernel, in the case of [20]) of the $S$-T-capitulation $\operatorname{map} j_{K / F, S}^{T}: C_{F, S}^{T} \rightarrow\left(C_{K, S}^{T}\right)^{G}$ for $T$-tamely ramified and $S$-split abelian extensions $K / F$ (see [19, 20] and [11, Corollary II.5.8.6, p.170]). Here $S$ denotes a finite set of primes of $F$, which we always assume to contain all archimedean primes in the number field case, and $T$ is a finite set of non-archimedean primes of $F$ which is disjoint from $S$. One of the few exceptions to this "general rule" is the work of B.Schmithals [23], who studied the kernel of $j_{K / F}$ for certain types of possibly ramified cyclic extensions $K$ of a quadratic number field $F$. However, the general problem of studying both the kernel and cokernel of $j_{K / F, S}^{T}$ for arbitrary finite Galois extensions $K$ of $F$ (i.e., not necessarily $S$-split or with ramification locus equal to $T$ ) has yet to be addressed. In particular, it is an unfortunate fact that, despite the long history of research on the Capitulation Problem, very little attention has been accorded the cokernel of $j_{K / F, S}^{T}$. In effect, apart from the contribution [20] already mentioned, the only other works known to us which study the capitulation cokernel are [9, Appendix] and, in the context of Iwasawa Theory, [13, 14, 15, 16, $18]$.

2000 Mathematics Subject Classification: 11R29, 11R27, 11R34.

Key words and phrases. Ideal class groups, capitulation, units in global fields, cohomology.

The author is partially supported by Fondecyt research grant 1061209 and Universidad Andrés Bello research grant DI-29-05/R. 
In this paper we study both the kernel and cokernel of the $S$-capitulation map $j_{K / F, S}: C_{F, S} \rightarrow C_{K, S}^{G}$ for arbitrary finite Galois extensions $K / F$ of global fields and arbitrary sets $S$ as above. We show, for example, that Ker $j_{K / F, S}$ may be identified with the subgroup of $H^{1}\left(G, U_{K, S}\right)$ of all cohomology classes which are locally trivial at all places outside $S$ (See Corollary 2.5$)^{1}$. We also obtain a new generalization of Hilbert's Theorem 94 (see Theorem 2.7), which applies to possibly ramified cyclic extensions of global fields (it is an open question to determine whether Theorem 2.7 below holds true for arbitrary abelian extensions, i.e., whether there exists a "ramified version" of Suzuki's Theorem [25]). In Section 3 we obtain certain general results on Coker $j_{K / F, S}$ which have some rather interesting consequences. For example, Theorem 3.7 states that in the "semisimple case" (i.e., when the degree of $K / F$ is prime to the class number $h_{K, S}$ ) it is possible to determine the structure of $H^{2}\left(G, U_{K, S}\right)$ completely. Sections 4-8 give applications of the main results of the paper. Theorem 4.1 states (roughly) that the structure of $H^{1}\left(G, U_{K}\right)$ is determined by that of $C_{F}$ and by the ramification indices of $K / F$ if $F$ belongs to a certain class of number fields and $K$ is equal to its own genus field relative to $F$ (the proof of this result uses well-known theorems of Tannaka-Terada, H.Furuya and C.Thiébaud). Section 5 deals with cyclic extensions and gives, under a certain hypothesis, a lower bound for the number of ambiguous $S$-classes of $K$ which do not come from $F$. See Theorem 5.2. This result may be regarded as a "dual" of Hilbert's Theorem 94. The very brief Section 6 computes the kernel and cokernel of the capitulation map for certain types of imaginary extensions of function fields. An application to imaginary Artin-Schreir extensions is given. Section 7 discusses the case where $S$ is large relative to $K / F$, i.e., when $S$ contains all archimedean primes and all ramified primes of $K / F$ (we note that a significant portion of earlier work on the Capitulation Problem has taken place in this setting). We show that in this case the kernel of the capitulation map is naturally isomorphic to $H^{1}\left(G, U_{K, S}\right)$, and that its cokernel is a certain group which measures the failure of the Hasse principle for $H^{2}\left(G, U_{K, S}\right)$. In Section 8, which concludes the paper, we use results from [22] to show that the main theorems of Sections 2 and 3 have natural analogs in the context of divisor class groups. This Section also contains some results which supplement those of [22]. Finally, the paper contains an Appendix which relates certain integers that appear in the main text to the ramification indices of $K / F$.

Allow us to make here the following additional comments which may help clarify the approach adopted in this paper. Let the global field $F$ be given and let $S$ and $T$ be given finite sets of primes of $F$ satisfying the conditions stated above. Then a natural question is to study the $S-T$ capitulation map $j_{K / F, S}^{T}$ for varying choices of $K$. If $K$ is chosen so that all primes of $S$ split completely in $K$ and $K / F$ is (tamely) ramified exactly over $T$, then we are in the setting of [20]. But other choices of $K$ are possible. For example, $K$ could be chosen so that the set of primes of $F$ which ramify in $K$ is in fact disjoint from $T$ (which is certainly the case in this paper since here we consider $T=\emptyset$ ), but no conditions are imposed regarding the behavior in $K$ of the primes in $S$. By adopting this approach, we have been able to obtain results on the cohomology of the units whenever information on Capitulation is available (see, e.g., Theorem 4.1), and results on Capitulation whenever information on the units is available (see, e.g., Theorem 5.2 and Example 5.3).

One final comment is in order. Several of the results of this paper immediately

${ }^{1}$ This result may be well-known to all specialists in this area. See Remark 2.6. 
yield divisibility relations which involve the class numbers $h_{F, S}, h_{K, S}$ and various other invariants (see, e.g., Theorem 5.2). Since explicitly stating all such divisibility relations would soon become quite repetitive, we have decided not to state them at all. We are certain that the interested readers will retrieve them without difficulty from the corresponding statements (for an illustration, see Example 5.3).

\section{ACKNOWLEDGEMENTS}

I am deeply grateful to D.Lorenzini for sending me copies of several of the references listed at the end of the paper, which would otherwise have been unavailable to me. I also thank C.Maire for sending me a copy of his thesis [19].

\section{Settings And notations.}

Let $F$ be a global field, i.e. $F$ is a finite extension of $\mathbb{Q}$ (the "number field case") or is finitely generated and of transcendence degree 1 over a finite field of constants $k$ of characteristic $p$ and cardinality $q$ (the "function field case"). Let $K / F$ be a finite Galois extension of global fields with Galois group $G$. In the function field case, we will write $k^{\prime}$ for the field of constants of $K$ and $q^{\prime}$ for its cardinality. The infinite primes of a function field $F$ are the primes which lie above the prime of $k(t)$ corresponding to the pole of $t$. A function field having only one infinite prime is called imaginary. Now let $S$ be any nonempty finite set of primes of the global field $F$, containing the archimedean primes in the number field case. Where confusion is unlikely, we will denote by $S$ (also) the set of primes of $K$ which lie above the primes in $S$. In the remaining instances, this set will be denoted by $S_{K}$. The set of non-archimedean primes of $F$ which ramify in $K$ will be denoted by $R$. Now, for each prime $v \in R \cup S$, we fix once and for all a prime $w$ of $K$ lying above $v$. We will write $\mathcal{I}_{F, S}$ for the group of fractional ideals (or divisors) of $F$ with support outside $S$. A similar notation will apply to $K$. Finally, the set of archimedean primes of a number field $F$ will be denoted by $S_{\infty}$.

\section{The CAPitUlation KeRnEL.}

We begin by considering the exact sequence of $G$-modules

$$
1 \rightarrow U_{K, S} \rightarrow K^{*} \rightarrow \mathcal{I}_{K, S} \rightarrow C_{K, S} \rightarrow 0,
$$

which we split into two short exact sequences of $G$-modules as follows:

$$
1 \rightarrow U_{K, S} \rightarrow K^{*} \rightarrow K^{*} / U_{K, S} \rightarrow 1
$$

and

$$
1 \rightarrow K^{*} / U_{K, S} \rightarrow \mathcal{I}_{K, S} \rightarrow C_{K, S} \rightarrow 0 .
$$

Lemma 2.1. We have

$$
H^{1}\left(G, \mathcal{I}_{K, S}\right)=0 .
$$

Proof. This is well-known. See, for example, [28, Lemma 2.1]. 
Set $\operatorname{Br}(K / F)=H^{2}\left(G, K^{*}\right)=\operatorname{Ker}\left[\operatorname{Br} F \rightarrow(\operatorname{Br} K)^{G}\right]$. By the preceding lemma and Hilbert's Theorem 90, (1) and (2) yield the following exact sequences:

$$
\begin{aligned}
1 & \rightarrow F^{*} / U_{F, S} \rightarrow\left(K^{*} / U_{K, S}\right)^{G} \rightarrow H^{1}\left(G, U_{K, S}\right) \rightarrow 0, \\
0 & \rightarrow H^{1}\left(G, K^{*} / U_{K, S}\right) \rightarrow H^{2}\left(G, U_{K, S}\right) \rightarrow \operatorname{Br}(K / F) \\
& \rightarrow H^{2}\left(G, K^{*} / U_{K, S}\right) \rightarrow H^{3}\left(G, U_{K, S}\right), \\
& 1 \rightarrow\left(K^{*} / U_{K, S}\right)^{G} \rightarrow \mathcal{I}_{K, S}^{G} \rightarrow\left(C_{K, S}\right)_{\text {trans }}^{G} \rightarrow 0, \\
0 & \rightarrow\left(C_{K, S}\right)_{\text {trans }}^{G} \rightarrow C_{K, S}^{G} \rightarrow H^{1}\left(G, K^{*} / U_{K, S}\right) \rightarrow 0,
\end{aligned}
$$

and

$$
0 \rightarrow H^{1}\left(G, C_{K, S}\right) \rightarrow H^{2}\left(G, K^{*} / U_{K, S}\right) \rightarrow H^{2}\left(G, \mathcal{I}_{K, S}\right),
$$

where, by definition ${ }^{2}$,

$$
\left(C_{K, S}\right)_{\text {trans }}^{G}=\operatorname{Ker}\left[C_{K, S}^{G} \rightarrow H^{1}\left(G, K^{*} / U_{K, S}\right)\right] .
$$

Note that, by $(5),\left(C_{K, S}\right)_{\text {trans }}^{G}$ is trivial exactly when every ambiguous $S$-ideal of $K$ is principal.

For subsequent use, we note that the connecting homomorphism $\left(K^{*} / U_{K, S}\right)^{G} \rightarrow$ $H^{1}\left(G, U_{K, S}\right)$ appearing in (3) maps a class $\beta U_{K, S} \in\left(K^{*} / U_{K, S}\right)^{G}$ to the cohomology class $\left\{\xi_{\beta}\right\} \in H^{1}\left(G, U_{K, S}\right)$ which is represented by the 1-cocycle $\xi_{\beta}: G \rightarrow U_{K, S}$ defined by $\xi_{\beta}(\sigma)=\beta^{\sigma} / \beta \quad(\sigma \in G)$.

Now let $j_{K / F, S}: C_{F, S} \rightarrow C_{K, S}^{G}$ be the natural capitulation map. It is not difficult to see, using the general description of the connecting homomorphism $C_{K, S}^{G} \rightarrow$ $H^{1}\left(G, K^{*} / U_{K, S}\right)$ appearing in (6) (see, e.g., [2, p.97]), that the image of $j_{K / F, S}$ is contained in $\left(C_{K, S}\right)_{\text {trans }}^{G}$. We will write $j_{K / F, S}^{\prime}: C_{F, S} \rightarrow\left(C_{K, S}\right)_{\text {trans }}^{G}$ for the map induced by $j_{K / F, S}$. Clearly, $\operatorname{Ker} j_{K / F, S}^{\prime}=\operatorname{Ker} j_{K / F, S}$ and (6) immediately yields the following proposition.

Proposition 2.2. There exists a natural exact sequence

$$
0 \rightarrow \operatorname{Coker} j_{K / F, S}^{\prime} \rightarrow \operatorname{Coker} j_{K / F, S} \rightarrow H^{1}\left(G, K^{*} / U_{K, S}\right) \rightarrow 0 .
$$

Now, we have a natural exact commutative diagram

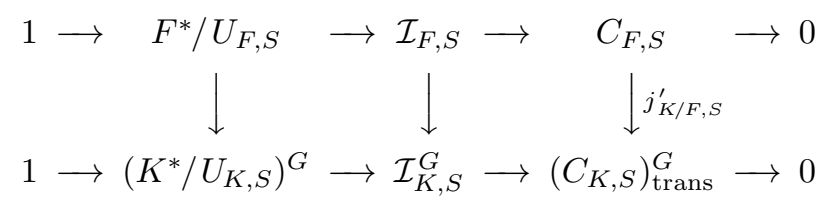

where the top row is the exact sequence (2) over $F$, the bottom row is (5), and the left vertical map comes from (3). The middle vertical map is injective and its cokernel has the following well-known description.

\footnotetext{
${ }^{2}$ The composite $C_{K, S}^{G} \rightarrow H^{1}\left(G, K^{*} / U_{K, S}\right) \hookrightarrow H^{2}\left(G, U_{K, S}\right)$ is known as the transgression map, and $\left(C_{K, S}\right)_{\text {trans }}^{G}$ might well be called the group of transgressive ambiguous classes. However, $\left(C_{K, S}\right)_{\text {trans }}^{G}$ is better known as the group of strongly ambiguous classes.
} 
Lemma 2.3. There exists a canonical isomorphism

$$
\operatorname{Coker}\left[\mathcal{I}_{F, S} \rightarrow\left(\mathcal{I}_{K, S}\right)^{G}\right] \simeq \bigoplus_{v \in R \backslash S} H^{1}\left(G_{w}, U_{w}\right)
$$

Proof. We give a proof of this well-known result because we will need some of the maps defined below. Let $D=\sum_{v \notin S} \sum_{w \mid v} n_{w}(D) w \in \mathcal{I}_{K, S}$. Since $G$ permutes transitively the primes of $K$ lying above the same prime of $F$, we have $D \in\left(\mathcal{I}_{K, S}\right)^{G}$ if and only if, for every $v \notin S$, the coefficients $n_{w}(D)$ coincide for all $w \mid v$. Write $n_{v}(D)$ for this common value. Then $D$ belongs to the image of the map $\mathcal{I}_{F, S} \rightarrow$ $\mathcal{I}_{K, S}, \sum m_{v} v \mapsto \sum m_{v} e_{v} \sum_{w \mid v} w$, if and only if $n_{v}(D)$ is divisible by $e_{v}$ for each $v \notin S$. Thus there exists a canonical isomorphism

$$
\operatorname{Coker}\left[\mathcal{I}_{F, S} \rightarrow\left(\mathcal{I}_{K, S}\right)^{G}\right] \simeq \bigoplus_{v \in R \backslash S} \mathbb{Z} / e_{v} \mathbb{Z}
$$

which maps the class of $D \in\left(\mathcal{I}_{K, S}\right)^{G}$ to $\left(n_{v}(D) \bmod e_{v}\right)_{v \in R \backslash S} \in \bigoplus_{v \in R \backslash S} \mathbb{Z} / e_{v} \mathbb{Z}$. On the other hand, for each $w \notin S_{K}$, there exists a natural exact sequence

$$
1 \rightarrow U_{w} \rightarrow K_{w}^{*} \stackrel{\operatorname{ord}_{w}}{\longrightarrow} \mathbb{Z} \rightarrow 0,
$$

where the map $\operatorname{ord}_{w}$ assigns the value 1 to a fixed uniformizing parameter of $K_{w}$. Let $v$ be the prime of $F$ lying below $w$ and write $G_{w}=\operatorname{Gal}\left(K_{w} / F_{v}\right)$. We have a natural exact commutative diagram

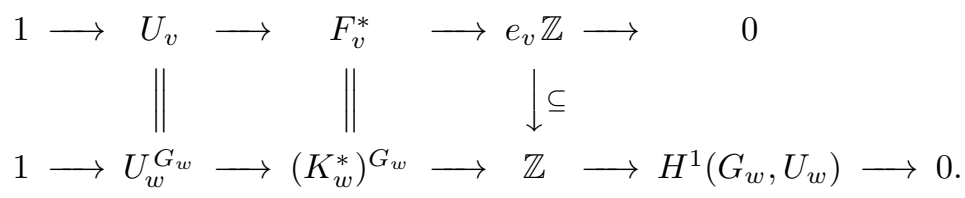

Thus we have a canonical isomorphism

$$
\mathbb{Z} / e_{v} \mathbb{Z} \simeq H^{1}\left(G_{w}, U_{w}\right)
$$

which maps a class $m$ mod $e_{v} \in \mathbb{Z} / e_{v} \mathbb{Z}$ to the cohomology class $\left\{\xi_{m}\right\} \in H^{1}\left(G_{w}, U_{w}\right)$ represented by the 1 -cocycle $\xi_{m}: G_{w} \rightarrow U_{w}$ given by $\xi_{m}(\sigma)=\beta^{\sigma} / \beta \quad\left(\sigma \in G_{w}\right)$,

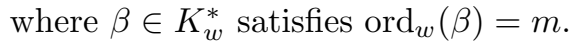

We now apply the snake lemma to diagram (8) and obtain an exact sequence

$$
\begin{aligned}
0 & \rightarrow \operatorname{Ker} j_{K / F, S} \rightarrow \operatorname{Coker}\left[F^{*} / U_{F, S} \rightarrow\left(K^{*} / U_{K, S}\right)^{G}\right] \stackrel{l}{\longrightarrow} \operatorname{Coker}\left[\mathcal{I}_{F, S} \rightarrow\left(\mathcal{I}_{K, S}\right)^{G}\right] \\
& \rightarrow \operatorname{Coker} j_{K / F, S}^{\prime} \rightarrow 0,
\end{aligned}
$$

where the map $l$ is induced by the natural map $K^{*} / U_{K, S} \rightarrow \mathcal{I}_{K, S}$. This map fits into a commutative diagram

$$
\begin{aligned}
& \operatorname{Coker}\left[F^{*} / U_{F, S} \rightarrow\left(K^{*} / U_{K, S}\right)^{G}\right] \stackrel{l}{\longrightarrow} \operatorname{Coker}\left[\mathcal{I}_{F, S} \rightarrow\left(\mathcal{I}_{K, S}\right)^{G}\right] \\
& \downarrow \simeq \quad \downarrow \simeq \\
& H^{1}\left(G, U_{K, S}\right) \quad \stackrel{\lambda}{\longrightarrow} \bigoplus_{v \in R \backslash S} H^{1}\left(G_{w}, U_{w}\right),
\end{aligned}
$$


where the right vertical map is the isomorphism of Lemma 2.3, the left vertical map is induced by the connecting homomorphism $\left(K^{*} / U_{K, S}\right)^{G} \rightarrow H^{1}\left(G, U_{K, S}\right)$ described earlier, and the bottom ("localization") map $\lambda$ may be described as follows: let $c \in H^{1}\left(G, U_{K, S}\right)$ be represented by the 1-cocycle $\xi: G \rightarrow U_{K, S}, \sigma \mapsto \beta^{\sigma} / \beta$, where $\beta U_{K, S} \in\left(K^{*} / U_{K, S}\right)^{G}$. Then the $v$-component of $\lambda(c)(v \in R \backslash S)$ is the cohomology class in $H^{1}\left(G_{w}, U_{w}\right)$ represented by the 1 -cocycle $\xi_{v}: G_{w} \rightarrow U_{w}$ given by $\xi_{v}(\sigma)=\beta^{\sigma} / \beta\left(\sigma \in G_{w}\right)$.

The above argument yields the following result.

Theorem 2.4. There exists an exact sequence

$$
0 \rightarrow \operatorname{Ker} j_{K / F, S} \rightarrow H^{1}\left(G, U_{K, S}\right) \stackrel{\lambda}{\longrightarrow} \bigoplus_{v \in R \backslash S} H^{1}\left(G_{w}, U_{w}\right) \rightarrow \operatorname{Coker} j_{K / F, S}^{\prime} \rightarrow 0
$$

where $j_{K / F, S}$ is the capitulation map, $j_{K / F, S}^{\prime}: C_{F} \rightarrow\left(C_{K, S}\right)_{\operatorname{trans}}^{G}$ is induced by $j_{K / F, S}$ and $\lambda$ is the localization map described above.

By the description of the map $\lambda$ given above and the proof of Lemma 2.3, the following is an immediate consequence of the theorem.

Corollary 2.5. The capitulation kernel $\operatorname{Ker} j_{K / F, S}$ is canonically isomorphic to the subgroup of $H^{1}\left(G, U_{K, S}\right)$ of all cohomology classes which are represented by a 1-cocycle $\xi: G \rightarrow U_{K, S}$ of the form $\xi(\sigma)=\beta^{\sigma} / \beta(\sigma \in G)$, where $\beta U_{K, S} \in$ $\left(K^{*} / U_{K, S}\right)^{G}$ satisfies $\operatorname{ord}_{w}(\beta) \equiv 0\left(\bmod e_{v}\right)$ for all $v \in R \backslash S$.

Remark 2.6. An equivalent form of the exact sequence of Theorem 2.4 was previously obtained by H.van der Wall [28, proof of Theorem 1.3, bottom of p.7] in the case that $F$ is a number field and $S$ is the set of all archimedean primes of $F$. See also [23, Theorem 2, p.46]. Further, we invite the reader to compare Corollary 2.5 (for number fields and $S=S_{\infty}$ ) with [23, Corollary, p.46].

Now set

$$
S^{\prime}=S \cup(R \backslash S)=S \cup R
$$

We define integers $d_{v}$, for $v \in S^{\prime}$, when $K / F$ is a cyclic extension of degree $n$ as follows:

$$
d_{v}=\left\{\begin{array}{cl}
{\left[K_{w}: F_{v}\right]} & \text { if } v \in S \\
e_{v} & \text { if } v \in R \backslash S .
\end{array}\right.
$$

Clearly, each $d_{v}$ is a divisor of $n$. Set

$$
D=\text { l.c.m. }\left\{d_{v}: v \in S^{\prime}\right\}
$$

and

$$
n_{0}=n / D
$$

Then we have the following generalization of Hilbert's Theorem 94. 
Theorem 2.7. Let $F$ be a global field and let $K / F$ be a cyclic extension of degree $n$. Let $d_{v}, D$ and $n_{0}$ be the integers (9), (10) and (11), respectively. Then at least $n_{0} /\left(n_{0}, \prod_{v \in S^{\prime}} d_{v} / D\right) S$-ideal classes of $F$ capitulate in $K$.

Proof. The proof of Lemma 2.3 and Theorem 2.4 immediately yield the order relation

$$
\left[\operatorname{Ker} j_{K / F, S}\right] \prod_{v \in R \backslash S} e_{v}=\left[H^{1}\left(G, U_{K, S}\right)\right]\left[\operatorname{Coker} j_{K / F, S}^{\prime}\right] .
$$

On the other hand, the well-known formula for the Herbrand quotient of the $G$ module $U_{K, S}$ (see, e.g., [26, Proof of Theorem 8.3, p.178]) yields the identity

$$
n\left[\widehat{H}^{0}\left(G, U_{K, S}\right)\right]=\left[H^{1}\left(G, U_{K, S}\right)\right] \prod_{v \in S}\left[K_{w}: F_{v}\right] .
$$

Combining the preceding formulas, we obtain the equality

$$
\frac{n}{\left(n, \prod_{v \in S^{\prime}} d_{v}\right)}\left[\widehat{H}^{0}\left(G, U_{K, S}\right)\right]\left[\operatorname{Coker} j_{K / F, S}^{\prime}\right]=\frac{\prod_{v \in S^{\prime}} d_{v}}{\left(n, \prod_{v \in S^{\prime}} d_{v}\right)}\left[\operatorname{Ker} j_{K / F, S}\right] .
$$

It follows that $n /\left(n, \prod_{v \in S^{\prime}} d_{v}\right)=n_{0} /\left(n_{0}, \prod_{v \in S^{\prime}} d_{v} / D\right)$ divides $\left[\operatorname{Ker} j_{K / F, S}\right]$, as asserted.

The group Coker $j_{K / F, S}^{\prime}$ appears to be as difficult to compute as Ker $j_{K / F, S}{ }^{3}$. We close this section by giving an alternative description of Coker $j_{K / F, S}^{\prime}$ (see Proposition 2.9 below) which may prove useful in future research on this group. We need the following approximation lemma.

Lemma 2.8. The natural map

$$
F^{*} / U_{F, S} \rightarrow \bigoplus_{v \in R \backslash S} F_{v}^{*} / U_{v}
$$

is surjective.

Proof. For each $v \in R \backslash S$, let $x_{v} U_{v} \in F_{v}^{*} / U_{v}$ and set $m=\max \left\{\operatorname{ord}_{v}\left(x_{v}\right): v \in\right.$ $R \backslash S\}$. By the strong approximation theorem [2, p.67], there exists a $\beta \in F^{*}$ such that $\operatorname{ord}_{v}\left(\beta-x_{v}\right)>m$ for all $v \in R \backslash S$. It follows that $\operatorname{ord}_{v}(\beta)=\operatorname{ord}_{v}\left(x_{v}\right)$ for every $v \in R \backslash S$, i.e., $\beta x_{v}^{-1} \in U_{v}$ for every $v \in R \backslash S$. Consequently $\beta U_{F, S} \in F^{*} / U_{F, S}$ maps to $\left(x_{v} U_{v}\right)_{v \in R \backslash S} \in \bigoplus_{v \in R \backslash S} F_{v}^{*} / U_{v}$.

We now consider the exact commutative diagram

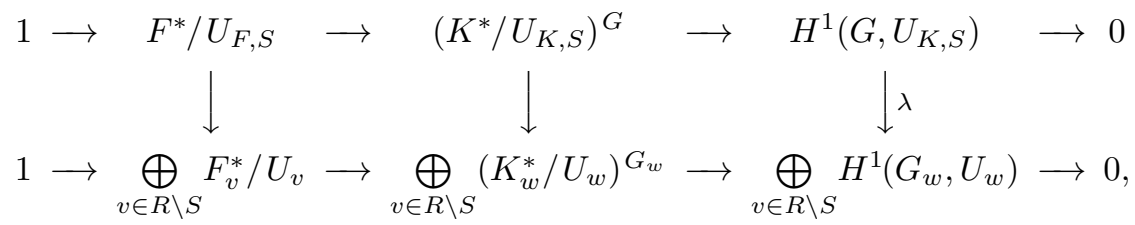

where the top row is the exact sequence (3), the bottom row is the direct sum over $v \in R \backslash S$ of exact sequences analogous to (3) for the extension $K_{w} / F_{v}$, and the

\footnotetext{
${ }^{3}$ See $[28]$ for the computation of $\left[\right.$ Coker $\left.j_{K / F, S}^{\prime}\right]$ when $S=S_{\infty}$ in some particular cases, notably when $K / F$ is an extension of prime degree of a quadratic number field $F$.
} 
unlabeled vertical maps are the natural ones. By the preceding lemma, the above diagram yields an isomorphism

$$
\text { Coker } \lambda \simeq \text { Coker }\left[\left(K^{*} / U_{K, S}\right)^{G} \rightarrow \bigoplus_{v \in R \backslash S}\left(K_{w}^{*} / U_{w}\right)^{G_{w}}\right] .
$$

Let

$$
\varphi:\left(K^{*} / U_{K, S}\right)^{G} \rightarrow \bigoplus_{v \in R \backslash S} \mathbb{Z}
$$

be given by

$$
\varphi\left(x U_{K, S}\right)=\left(\operatorname{ord}_{w}(x)\right)_{v \in R \backslash S} \quad\left(x \in K^{*}\right),
$$

i.e., $\varphi$ is the composite of the natural map $\left(K^{*} / U_{K, S}\right)^{G} \rightarrow \bigoplus_{v \in R \backslash S}\left(K_{w}^{*} / U_{w}\right)^{G_{w}}$ and the isomorphism $\bigoplus \operatorname{ord}_{w}: \bigoplus_{v \in R \backslash S}\left(K_{w}^{*} / U_{w}\right)^{G_{w}} \stackrel{\sim}{\longrightarrow} \bigoplus_{v \in R \backslash S} \mathbb{Z}$. Then (12) induces an isomorphism

$$
\text { Coker } \lambda \simeq \operatorname{Coker} \varphi
$$

On the other hand, Theorem 2.4 yields an isomorphism Coker $j_{K / F, S}^{\prime} \simeq$ Coker $\lambda$. Thus the following holds.

Proposition 2.9. There exists an isomorphism

$$
\text { Coker } j_{K / F, S}^{\prime} \simeq \operatorname{Coker} \varphi
$$

where $\varphi$ is the map (13).

\section{The CApitulation COKERNel.}

We now recall the exact sequence (4):

$$
\begin{aligned}
0 & \rightarrow H^{1}\left(G, K^{*} / U_{K, S}\right) \rightarrow H^{2}\left(G, U_{K, S}\right) \rightarrow \operatorname{Br}(K / F) \rightarrow H^{2}\left(G, K^{*} / U_{K, S}\right) \\
& \rightarrow H^{3}\left(G, U_{K, S}\right) .
\end{aligned}
$$

We note that the map $H^{2}\left(G, U_{K, S}\right) \rightarrow \operatorname{Br}(K / F)=H^{2}\left(G, K^{*}\right)$ appearing above is induced by the inclusion $U_{K, S} \subset K^{*}$. Now let

$$
\mathrm{B}\left(\mathcal{O}_{F, S}, \mathcal{O}_{K, S}\right)=\operatorname{Ker}\left[\operatorname{Br}(K / F) \rightarrow H^{2}\left(G, \mathcal{I}_{K, S}\right)\right]
$$

where the map involved is induced by the natural map $K^{*} \rightarrow \mathcal{I}_{K, S}, x \rightarrow(x)$. Then, by (7), the above exact sequence induces an exact sequence ${ }^{4}$

$$
\begin{aligned}
0 & \rightarrow H^{1}\left(G, K^{*} / U_{K, S}\right) \rightarrow H^{2}\left(G, U_{K, S}\right) \rightarrow \mathrm{B}\left(\mathcal{O}_{F, S}, \mathcal{O}_{K, S}\right) \rightarrow H^{1}\left(G, C_{K, S}\right) \\
& \rightarrow H^{3}\left(G, U_{K, S}\right) .
\end{aligned}
$$

See $[16, \S 1]$ for the details. By combining the preceding exact sequence with Proposition 2.2, we obtain the following result.

Proposition 3.1. There exists a natural six-term exact sequence

$$
\begin{aligned}
0 & \rightarrow \text { Coker } j_{K / F, S}^{\prime} \rightarrow \text { Coker } j_{K / F, S} \rightarrow H^{2}\left(G, U_{K, S}\right) \rightarrow \mathrm{B}\left(\mathcal{O}_{F, S}, \mathcal{O}_{K, S}\right) \\
& \rightarrow H^{1}\left(G, C_{K, S}\right) \rightarrow H^{3}\left(G, U_{K, S}\right),
\end{aligned}
$$

where $\mathrm{B}\left(\mathcal{O}_{F, S}, \mathcal{O}_{K, S}\right)$ is the group (14).

\footnotetext{
${ }^{4}$ This exact sequence was discovered independently by M.Auslander and A.Brumer in [3] and by S.Chase, D.Harrison and A.Rosenberg in [6]. It was used in [5] and in [16]. More recently, it resurfaced in [9, Appendix], where a particular case of (15) was derived from a Hochschild-Serre spectral sequence in étale cohomology.
} 
We will now use class field theory to compute $\mathrm{B}\left(\mathcal{O}_{F, S}, \mathcal{O}_{K, S}\right)$. This computation generalizes [9, Lemma A.1].

By global class field theory, $\mathrm{Br} F$ is naturally isomorphic to the kernel of the map $\sum \operatorname{inv}_{v}: \bigoplus_{\text {all } v} \operatorname{Br} F_{v} \rightarrow \mathbb{Q} / \mathbb{Z}$. Under this isomorphism, the subgroup $\operatorname{Br}(K / F)$ of $\mathrm{Br} F$ may be identified with the kernel of the induced map

$$
\sum \operatorname{inv}_{v}: \bigoplus_{\text {all } v} H^{2}\left(G_{w}, K_{w}^{*}\right) \rightarrow \mathbb{Q} / \mathbb{Z}
$$

where, for each $v$, we regard $H^{2}\left(G_{w}, K_{w}^{*}\right)$ as a subgroup of $\mathrm{Br} F_{v}$ via the inflation map $\operatorname{Inf}_{w / v}: H^{2}\left(G_{w}, K_{w}^{*}\right) \rightarrow \operatorname{Br}\left(F_{v}\right)$ (which is injective). On the other hand, there exist well-known canonical isomorphisms

$$
\begin{aligned}
H^{2}\left(G, \mathcal{I}_{K, S}\right) & \simeq \bigoplus_{v \notin S} H^{2}\left(G_{w},\left\langle\mathfrak{p}_{w}\right\rangle\right) \simeq \bigoplus_{v \notin S} H^{2}\left(G_{w}, \mathbb{Z}\right) \\
& \simeq \bigoplus_{v \notin S} H^{2}\left(G_{w}, K_{w}^{*} / U_{w}\right) .
\end{aligned}
$$

Let $\psi: H^{2}\left(G, \mathcal{I}_{K, S}\right) \rightarrow \bigoplus_{v \notin S} H^{2}\left(G_{w}, K_{w}^{*} / U_{w}\right)$ be the composite of the above isomorphisms. Then the diagram

$$
\begin{aligned}
& \operatorname{Br}(K / F) \longrightarrow\left(\bigoplus_{v \in S} H^{2}\left(G_{w}, K_{w}^{*}\right)\right) \oplus\left(\bigoplus_{v \notin S} H^{2}\left(G_{w}, K_{w}^{*}\right)\right) \\
& \downarrow \downarrow \downarrow\left(f_{1}, \oplus_{v \notin S} f_{2, v}\right) \\
& H^{2}\left(G, \mathcal{I}_{K, S}\right) \stackrel{\psi}{\longrightarrow} \quad \bigoplus_{v \notin S} H^{2}\left(G_{w}, K_{w}^{*} / U_{w}\right),
\end{aligned}
$$

where $f_{1}$ is the zero map and $f_{2, v}: H^{2}\left(G_{w}, K_{w}^{*}\right) \rightarrow H^{2}\left(G_{w}, K_{w}^{*} / U_{w}\right)$, for each $v \notin S$, is induced by the natural map $K_{w}^{*} \rightarrow K_{w}^{*} / U_{w}$, commutes. It follows that the identification

$$
\operatorname{Br}(K / F)=\operatorname{Ker}\left[\bigoplus_{\text {all } v} H^{2}\left(G_{w}, K_{w}^{*}\right) \stackrel{\sum \text { inv }_{v}}{\longrightarrow} \mathbb{Q} / \mathbb{Z}\right]
$$

induces an identification

$$
\mathrm{B}\left(\mathcal{O}_{F, S}, \mathcal{O}_{K, S}\right)=\operatorname{Ker}\left[\bigoplus_{v \in S} H^{2}\left(G_{w}, K_{w}^{*}\right) \oplus \bigoplus_{v \notin S} \operatorname{Ker} f_{2, v} \stackrel{\sum \operatorname{inv}_{v}}{\longrightarrow} \mathbb{Q} / \mathbb{Z}\right] .
$$

Now, since $H^{1}\left(G_{w}, K_{w}^{*} / U_{w}\right) \simeq H^{1}\left(G_{w}, \mathbb{Z}\right)=0$ for each $v \notin S$, there exist canonical isomorphisms

$$
\operatorname{Ker} f_{2, v} \simeq H^{2}\left(G_{w}, U_{w}\right) \quad(v \notin S) .
$$

Note that the latter group is zero if $v \notin R$. Now recall the set $S^{\prime}=S \cup(R \backslash S)=$ $S \cup R$. We define integers $d_{v}$, for $v \in S^{\prime}$, as follows ${ }^{5}$ :

$$
d_{v}=\left\{\begin{array}{cl}
{\left[K_{w}: F_{v}\right]} & \text { if } v \in S \\
{\left[H^{2}\left(G_{w}, U_{w}\right)\right]} & \text { if } v \in R \backslash S .
\end{array}\right.
$$
[1].

${ }^{5}$ If $K / F$ is cyclic, then the integers (18)-(19) agree with the integers (9)-(10). See, for example, 
Further, set

$$
D=\text { l.c.m. }\left\{d_{v}: v \in S^{\prime}\right\} \text {. }
$$

Then the invariant map $\operatorname{inv}_{v}$ induces isomorphisms

$$
\begin{aligned}
& H^{2}\left(G_{w}, K_{w}^{*}\right) \simeq d_{v}^{-1} \mathbb{Z} / \mathbb{Z} \quad(v \in S) \\
& H^{2}\left(G_{w}, U_{w}\right) \simeq d_{v}^{-1} \mathbb{Z} / \mathbb{Z} \quad(v \in R \backslash S) .
\end{aligned}
$$

It follows from (16), (17), (20) and (21) that there exists a natural isomorphism

$$
\mathrm{B}\left(\mathcal{O}_{F, S}, \mathcal{O}_{K, S}\right) \simeq \operatorname{Ker}\left[\bigoplus_{v \in S^{\prime}} d_{v}^{-1} \mathbb{Z} / \mathbb{Z} \stackrel{\Sigma}{\longrightarrow} D^{-1} \mathbb{Z} / \mathbb{Z}\right]
$$

where $\Sigma$ is the summation map $\left(x_{v}\right) \rightarrow \sum x_{v}$. The latter map is surjective (see [10, Lemma 1.2]), whence the following holds.

Lemma 3.2. There exists an exact sequence

$$
0 \rightarrow \mathrm{B}\left(\mathcal{O}_{F, S}, \mathcal{O}_{K, S}\right) \rightarrow \bigoplus_{v \in S^{\prime}} \mathbb{Z} / d_{v} \mathbb{Z} \stackrel{\Sigma}{\longrightarrow} \mathbb{Z} / D \mathbb{Z} \rightarrow 0
$$

where $d_{v}$ and $D$ are the integers (18) and (19), respectively. In particular,

$$
\left[\mathrm{B}\left(\mathcal{O}_{F, S}, \mathcal{O}_{K, S}\right)\right]=\left(\prod_{v \in S^{\prime}} d_{v}\right) / D
$$

Now, combining Proposition 3.1 and Lemma 3.2, we obtain the following result.

Theorem 3.3. There exists an exact sequence

$$
\begin{aligned}
0 & \rightarrow \text { Coker } j_{K / F, S}^{\prime} \rightarrow \text { Coker } j_{K / F, S} \rightarrow H^{2}\left(G, U_{K, S}\right) \rightarrow \mathrm{B}\left(\mathcal{O}_{F, S}, \mathcal{O}_{K, S}\right) \\
& \rightarrow H^{1}\left(G, C_{K, S}\right) \rightarrow H^{3}\left(G, U_{K, S}\right),
\end{aligned}
$$

where $\mathrm{B}\left(\mathcal{O}_{F, S}, \mathcal{O}_{K, S}\right)$ is a group of order $\left(\prod_{v \in S^{\prime}} d_{v}\right) / D$.

Remark 3.4. We note that, via the isomorphisms (16) and (17), the map $H^{2}\left(G, U_{K, S}\right) \rightarrow \mathrm{B}\left(\mathcal{O}_{F, S}, \mathcal{O}_{K, S}\right)$ appearing in the exact sequence of the theorem is induced by the inclusions $U_{K, S} \subset U_{w}(v \notin S)$ and $U_{K, S} \subset K_{w}^{*}(v \in S)$. Thus, by $(15)$,

$$
\begin{aligned}
H^{1}\left(G, K^{*} / U_{K, S}\right) & \simeq \operatorname{Ker}\left[H^{2}\left(G, U_{K, S}\right) \rightarrow \mathrm{B}\left(\mathcal{O}_{F, S}, \mathcal{O}_{K, S}\right)\right] \\
& \simeq \operatorname{Ker}\left[H^{2}\left(G, U_{K, S}\right) \rightarrow \bigoplus_{v \in R \backslash S} H^{2}\left(G_{w}, U_{w}\right) \oplus \bigoplus_{v \in S} H^{2}\left(G_{w}, K_{w}^{*}\right)\right],
\end{aligned}
$$

where the last map is induced by the inclusions $U_{K, S} \subset U_{w}(v \notin S)$ and $U_{K, S} \subset K_{w}^{*}$ $(v \in S)$.

We now derive some consequences of Theorem 3.3. The first one generalizes $[22$, Theorem 2(C), p.161] (see Proposition 2.2).

Corollary 3.5. Assume that the integers $d_{v}$, where $v \in S^{\prime}$, are pairwise coprime. Then there exists a natural exact sequence

$$
0 \rightarrow \text { Coker } j_{K / F, S}^{\prime} \rightarrow \text { Coker } j_{K / F, S} \rightarrow H^{2}\left(G, U_{K, S}\right) \rightarrow 0 .
$$

Corollary 3.6. Assume that $H^{2}\left(G, U_{K, S}\right)=0$. Then $H^{1}\left(G, C_{K, S}\right)$ contains at least $\left(\prod_{v \in S^{\prime}} d_{v}\right) / D$ elements. 
The next theorem refers to the "limit case" where $[K: F]$ is prime to $h_{K, S}{ }^{6}$.

Theorem 3.7. Let $K / F$ be a Galois extension of global fields, of degree n. Assume that $n$ is prime to $h_{K, S}$. Then there exists a natural isomorphism

$$
H^{2}\left(G, U_{K, S}\right) \simeq \operatorname{Ker}\left[\bigoplus_{v \in S^{\prime}} \mathbb{Z} / d_{v} \mathbb{Z} \stackrel{\Sigma}{\longrightarrow} \mathbb{Z} / D \mathbb{Z}\right]
$$

In particular,

$$
\left[H^{2}\left(G, U_{K, S}\right)\right]=\left(\prod_{v \in S^{\prime}} d_{v}\right) / D \text {. }
$$

Proof. Let $N: C_{K, S} \rightarrow C_{K, S}^{G}$ be the map $c \mapsto \prod_{\sigma \in G} c^{\sigma}$. Then $N=j_{K / F, S} \circ$ $N_{K / F}$, where $N_{K / F}: C_{K, S} \rightarrow C_{F, S}$ is induced by the norm of ideals. Consequently, Coker $j_{K / F, S}$ is a quotient of $C_{K, S}^{G} / N C_{K, S}=\widehat{H}^{0}\left(G, C_{K, S}\right)$. Since the latter group is annihilated by multiplication by $\left(n, h_{K, S}\right)=1$, we conclude that Coker $j_{K / F, S}=0$. The theorem now follows from Theorem 3.3 since $H^{1}\left(G, C_{K, S}\right)$ is also annihilated by multiplication by $\left(n, h_{K, S}\right)=1$.

Remark 3.8. If $K / F$ is cyclic of degree $n$, and $n$ is prime to $h_{K, S}$, then the order of Ker $j_{K / F, S}$ may be computed explicitly. Indeed, by the theorem, $\left[\widehat{H}^{0}\left(G, U_{K, S}\right)\right]=\left[H^{2}\left(G, U_{K, S}\right)\right]=\left(\prod_{v \in S^{\prime}} d_{v}\right) / D$. On the other hand, the proof of the above theorem and Proposition 2.2 show that Coker $j_{K / F, S}^{\prime}=0$. Finally the proof of Theorem 2.7 yields

$$
\left[\operatorname{Ker} j_{K / F, S}\right]=n_{0}
$$

where $n_{0}=n / D$.

\section{Genus Fields.}

In this section we consider abelian extensions of certain base fields $F$ which are equal to their own genus field relative to $F$. For such extensions (and a minimal set $S$ ), the group $\left(C_{K, S}\right)_{\text {trans }}^{G}$ is zero and the following holds.

Theorem 4.1. Let $K / F$ be a finite abelian extension of number fields. Assume that $K$ is its own genus field relative to $F$. Assume, furthermore, that one of the following conditions holds:

(a) $K / F$ is cyclic, or

(b) $F$ is either the rational field $\mathbb{Q}$ or an imaginary quadratic extension of $\mathbb{Q}$ of discriminant $<-4$ and conductor prime to 2.

Then there exists an exact sequence

$$
0 \rightarrow C_{F} \rightarrow H^{1}\left(G, U_{K}\right) \rightarrow \bigoplus_{v \text { non-arch. }} \mathbb{Z} / e_{v} \mathbb{Z} \rightarrow 0
$$

Furthermore, there exists a canonical isomorphism

$$
C_{K}^{G} \simeq H^{1}\left(G, K^{*} / U_{K, S}\right) .
$$

\footnotetext{
${ }^{6}$ This condition can always be satisfied by enlarging $S$ appropriately. We also note that this case has been previously studied by H.Yokoi in [29, §4].
} 
In particular, if $h_{F}=1$, there exists an isomorphism ${ }^{7}$

$$
H^{1}\left(G, U_{K}\right) \simeq \bigoplus_{v \text { non-arch. }} \mathbb{Z} / e_{v} \mathbb{Z}
$$

Proof. We apply results from the preceding sections with $S$ equal to the set of all archimedean primes of $F$. By theorems of Tannaka-Terada, H.Furuya [8] and C.Thiebaud [27], every ambiguous ideal of $K$ is principal. Therefore $\left(C_{K}\right)_{\text {trans }}^{G}=0$ (see (5)), whence $\operatorname{Ker} j_{K / F}=C_{F}$, Coker $j_{K / F}^{\prime}=0$ and Coker $j_{K / F}=C_{K}^{G}$. The theorem is now immediate from Theorem 2.4 and Proposition 2.2.

We note that the preceding theorem applies, in particular, to ray class fields over $F$ since such fields are equal to their own genus field relative to $F^{8}$.

Remark 4.2. There exists a function field analog of the preceding theorem. Indeed, let $F$ be a function field, let $\infty$ be a fixed place of $F$ and let $S=\{\infty\}$. Further, let $K$ be a finite abelian extension of $F$ where $\infty$ is tamely ramified and the decomposition and inertia groups of $\infty$ agree. These extensions were called "of type (*)" in [7]. Assume further that $K$ is its own $S$-genus field. Then the main theorem of [7], combined with (5), shows that $\left(C_{K, S}\right)_{\text {trans }}^{G}=0$. Consequently, Theorem 2.4 yields an exact sequence

$$
0 \rightarrow C_{F, S} \rightarrow H^{1}\left(G, U_{K, S}\right) \rightarrow \bigoplus_{v \neq \infty} \mathbb{Z} / e_{v} \mathbb{Z} \rightarrow 0
$$

In particular, if $h_{F, S}=1$, there exists an isomorphism

$$
H^{1}\left(G, U_{K, S}\right) \simeq \bigoplus_{v \neq \infty} \mathbb{Z} / e_{v} \mathbb{Z}
$$

The above generalizes [7, Corollary 4.3].

\section{Cyclic extensions.}

In this Section we assume that $K / F$ is a cyclic extension of degree $n$.

Set

$$
W_{F, S}=U_{F, S} \cap N_{K / F} K^{*} .
$$

Then, by (4) and the periodicity of the cohomology of cyclic groups, we have

$$
\begin{aligned}
H^{1}\left(G, K^{*} / U_{K, S}\right) & \simeq \operatorname{Ker}\left[H^{2}\left(G, U_{K, S}\right) \rightarrow H^{2}\left(G, K^{*}\right)\right] \\
& \simeq \operatorname{Ker}\left[U_{F, S} / N_{K / F} U_{K, S} \rightarrow F^{*} / N_{K / F} K^{*}\right] \\
& =W_{F, S} / N_{K / F} U_{K, S}
\end{aligned}
$$

(cf. [22, Theorem 2(B), p.161]). Thus, by Proposition 2.2, the following holds.

\footnotetext{
${ }^{7}$ More generally, if $C_{F}=C_{F}^{e_{v}}$ for every non-archimedean prime $v$, then $H^{1}\left(G, U_{K}\right) \simeq C_{F} \oplus$ $\left(\oplus_{v \text { non-arch. }} \mathbb{Z} / e_{v} \mathbb{Z}\right)$.

${ }^{8}$ See [27, Lemma 2.1] for a general description of the abelian extensions of $F$ which are equal to their own genus field relative to $F$.
} 
Lemma 5.1. Assume that $K / F$ is a cyclic extension of global fields. Then there exists a natural exact sequence

$$
0 \rightarrow \text { Coker } j_{K / F, S}^{\prime} \rightarrow \text { Coker } j_{K / F, S} \rightarrow W_{F, S} / N_{K / F} U_{K, S} \rightarrow 0,
$$

where $W_{F, S}$ is the group (22).

The next result may be regarded as a "dual" of Theorem 2.7.

Theorem 5.2. Let $K / F$ be a cyclic extension of global fields. Assume that $U_{F, S} \subset$ $N_{K / F} K^{*}$. Then Coker $j_{K / F, S}$ contains at least

$$
\frac{\prod_{v \in S^{\prime}} d_{v} / D}{\left(n_{0}, \prod_{v \in S^{\prime}} d_{v} / D\right)}
$$

classes, where $d_{v}, D$ and $n_{0}$ are the integers (9), (10) and (11), respectively.

Proof. The lemma and the proof of Theorem 2.7 immediately yield the formula

$$
\frac{n}{\left(n, \prod_{v \in S^{\prime}} d_{v}\right)}\left[U_{F, S}: W_{F, S}\right]\left[\text { Coker } j_{K / F, S}\right]=\frac{\prod_{v \in S^{\prime}} d_{v}}{\left(n, \prod_{v \in S^{\prime}} d_{v}\right)}\left[\operatorname{Ker} j_{K / F, S}\right] .
$$

Therefore

$$
\frac{\prod_{v \in S^{\prime}} d_{v}}{\left(n, \prod_{v \in S^{\prime}} d_{v}\right)}=\frac{\prod_{v \in S^{\prime}} d_{v} / D}{\left(n_{0}, \prod_{v \in S^{\prime}} d_{v} / D\right)}
$$

divides $\left[U_{F, S}: W_{F, S}\right]\left[\right.$ Coker $\left.j_{K / F, S}\right]$. The result is now clear since $W_{F, S}=U_{F, S}$ by hypothesis.

Example 5.3. Let $F$ be either $\mathbb{Q}$, an imaginary quadratic number field (with $S=S_{\infty}$ in both cases) or a function field with $\# S=1$. Let $K$ be a cyclic extension of $F$ of degree $l^{m}$, where $m$ is a positive integer and $l$ is a rational prime which is either $\geq 5$ in the number field case or prime to $q-1$ in the function field case. Then $l^{m}$ is prime to the order of the finite group $U_{F, S}$, whence $U_{F, S}=$ $N_{K / F} U_{K, S} \subset N_{K / F} K^{*}$. Thus the hypothesis of Theorem 5.2 is satisfied. Assume now, for simplicity, that in the function field case the prime in $S$ splits completely in $K$. Let $l^{t_{1}}, l^{t_{2}}, \ldots, l^{t_{r}}$ be the ramification indices of the ramified primes of $K / F$, and assume that $m<\sum_{i=1}^{r} t_{i}$. Then the theorem asserts that $l^{t} \mid\left[\operatorname{Coker} j_{K / F, S}\right]$, where

$$
t=\sum_{i=1}^{r} t_{i}-m
$$

In particular, if $K / k(t)$ is a real Artin-Schreir extension, i.e., $K=F(\alpha)$, where $\alpha$ is a root of $x^{p}-x+Q(t)=0$ and $Q(t)$ is such that the infinite prime of $k(t)$ splits in $K$, then

$$
\operatorname{rank}_{p}\left(C_{K}^{G}\right) \geq r-1,
$$

where $r$ is the number of finite primes of $k(t)$ which ramify in $K^{9}$.

\footnotetext{
${ }^{9}$ See Corollary 6.2 for the case of imaginary Artin-Schreir extensions.
} 
We conclude this Section by noting that (15) and the identifications

$$
H^{1}\left(G, K^{*} / U_{K, S}\right) \simeq W_{F, S} / N_{K / F} U_{K, S}
$$

and

$$
H^{2}\left(G, U_{K, S}\right) \simeq U_{F, S} / N_{K / F} U_{K, S}
$$

show that $U_{F, S} / W_{F, S}$ is isomorphic to a subgroup of $\mathrm{B}\left(\mathcal{O}_{F, S}, \mathcal{O}_{K, S}\right)$. Consequently, Lemma 3.2 yields the following generalization of [29, Theorem 1(iv)] (see also [op.cit., Lemma 5]).

Theorem 5.4. The index $\left[U_{F, S}: W_{F, S}\right]$ divides $\left(\prod_{v \in S^{\prime}} d_{v}\right) / D$. Consequently, if the integers $d_{v}$, for $v \in S^{\prime}$, are pairwise coprime, then every $S$-unit of $F$ is a norm from $K$.

Remark 5.5. Note that in the "limit case" $\left[U_{F, S}: W_{F, S}\right]=\left(\prod_{v \in S^{\prime}} d_{v}\right) / D$ (cf. Theorem 3.7), the proof of Theorem 5.2 yields the identity

$$
\left[\operatorname{Ker} j_{K / F, S}\right]=n_{0}\left[\text { Coker } j_{K / F, S}\right] \text {, }
$$

where $n_{0}=n / D$. In particular, Ker $j_{K / F, S}$ contains at least $n_{0}$ elements. Compare Remark 3.8.

\section{IMAGINARY EXTENSIONS OF FUNCTION FIELDS.}

The main result of this section is the following

Theorem 6.1. Let $F$ be a function field, let $K$ be a Galois extension of $F$ of degree $n$ and let $k^{\prime}$ be the field of constants of $K$. Assume that $\# S_{K}=1$ and that $n$ is prime to $q^{\prime}-1$, where $q^{\prime}$ is the cardinality of $k^{\prime}$. Then there exist an exact sequence

$$
0 \rightarrow C_{F, S} \rightarrow C_{K, S}^{G} \rightarrow \bigoplus_{R \backslash S} \mathbb{Z} / e_{v} \mathbb{Z} \rightarrow 0
$$

and an isomorphism

$$
H^{1}\left(G, C_{K, S}\right) \simeq \operatorname{Ker}\left[\bigoplus_{v \in S^{\prime}} \mathbb{Z} / d_{v} \mathbb{Z} \stackrel{\Sigma}{\longrightarrow} \mathbb{Z} / D \mathbb{Z}\right] .
$$

Proof. The hypothesis $\# S_{K}=1$ (i.e., $S$ consists of exactly one prime of $F$ and there is only one prime of $K$ lying above it) implies, by Dirichlet's Unit Theorem, that $U_{K, S}=\left(k^{\prime}\right)^{*}$. Hence $H^{i}\left(G, U_{K, S}\right)=H^{i}\left(G,\left(k^{\prime}\right)^{*}\right)$ is annihilated by multiplication by $\left(n, q^{\prime}-1\right)$ for all $i \geq 1$. As $\left(n, q^{\prime}-1\right)=1$ by hypothesis, we conclude that $H^{i}\left(G, U_{K, S}\right)=0$ for all $i \geq 1$. The theorem now follows by combining Theorems 2.4 and 3.3 .

The following result is one possible application of Theorem 6.1.

Corollary 6.2. Let $F=k(t)$ with $k$ a finite field of characteristic $p$, let $Q(t) \in F^{*}$ and let $K=F(\alpha)$, where $\alpha$ is a root of $x^{p}-x+Q(t)=0$. Assume that $K$ is imaginary, i.e., that there exists only one prime of $K$ lying above the infinite prime of $F$. Then there exists a canonical isomorphism

$$
C_{K}^{G} \simeq(\mathbb{Z} / p \mathbb{Z})^{r}
$$

where $r$ is the number of finite primes of $F$ which ramify in $K$.

Proof. Note that $K$ is a Galois extension of $F$ of degree $p$, which is prime to $q^{\prime}-1$. Further, $h_{F}=1$. The corollary is immediate from the theorem. 


\section{LARGE $S$.}

A (nonempty) set $S$ of primes of a global field $F$ is large relative to $K / F$ if $S$ contains all archimedean primes of $F$ and all primes that ramify in $K / F$. In this section we assume that our set $S$ is large. Note then that $S^{\prime}=S \cup(R \backslash S)=S$.

Theorem 7.1. Let $K / F$ be a finite Galois extension of global fields with Galois group $G$, and let $S$ be a set of primes of $F$ which is large relative to $K / F$ (as defined above). Then there exists an exact sequence

$$
0 \rightarrow H^{1}\left(G, U_{K, S}\right) \rightarrow C_{F, S} \rightarrow C_{K, S}^{G} \rightarrow \amalg^{2}\left(G, U_{K, S}\right) \rightarrow 0,
$$

where

$$
\amalg^{2}\left(G, U_{K, S}\right)=\operatorname{Ker}\left[H^{2}\left(G, U_{K, S}\right) \rightarrow \bigoplus_{v \in S} H^{2}\left(G_{w}, K_{w}^{*}\right)\right],
$$

the map involved being induced by the inclusions $U_{K, S} \subset K_{w}^{*}(v \in S)$.

Proof. Since $S \supset R$, Theorem 2.4 shows that $\operatorname{Ker} j_{K / F, S}=H^{1}\left(G, U_{K, S}\right)$ and that Coker $j_{K / F, S}^{\prime}=0$. Now Proposition 2.2 and Remark 3.4 yield Coker $j_{K / F, S}=$ $\amalg^{2}\left(G, U_{K, S}\right)$, where $\amalg^{2}\left(G, U_{K, S}\right)$ is as in the statement.

The following corollary of the above theorem should be compared with [9, Proposition A.2].

Corollary 7.2. Let $K / F$ be a finite Galois extension of global fields with Galois group $G$. Assume that

(a) exactly one prime $v_{0}$ of $F$ ramifies in $K$,

(b) $S \supset S_{\infty} \cup\left\{v_{0}\right\}$ (i.e., $S$ is large relative to $K / F$ ), and

(c) every prime in $S \backslash\left\{v_{0}\right\}$ splits completely in $K$.

Then there exists an exact sequence

$$
0 \rightarrow H^{1}\left(G, U_{K, S}\right) \rightarrow C_{F, S} \rightarrow C_{K, S}^{G} \rightarrow H^{2}\left(G, U_{K, S}\right) \rightarrow 0 .
$$

Proof. By Lemma 3.2, $\mathrm{B}\left(\mathcal{O}_{F, S}, \mathcal{O}_{K, S}\right)$ is a group of order

$$
\left(\prod_{v \in S}\left[K_{w}: F_{v}\right]\right) / \text { l.c.m. }\left\{\left[K_{w}: F_{v}\right]: v \in S\right\}=\left[K_{w_{0}}: F_{v_{0}}\right] /\left[K_{w_{0}}: F_{v_{0}}\right]=1 .
$$

Consequently

$$
\amalg^{2}\left(G, U_{K, S}\right)=\operatorname{Ker}\left[H^{2}\left(G, U_{K, S}\right) \rightarrow \mathrm{B}\left(\mathcal{O}_{F, S}, \mathcal{O}_{K, S}\right)\right]=H^{2}\left(G, U_{K, S}\right)
$$

(see Remark 3.4). The corollary is now immediate from the theorem.

\section{Divisor Class Groups.}

This Section may be regarded as the " $S=\emptyset$ " version of Sections 2 and 3 in the function field case. We follow [22] closely. 
Let $K / F$ be a Galois extension of degree $n$ of function fields with Galois group $G$. Let $X^{\prime}$ denote the unique smooth complete curve over $k^{\prime}$ with function field $K$. Similarly, let $X$ be the smooth complete curve over $k$ with function field $F$. Further, let $H=\operatorname{Gal}\left(K / F k^{\prime}\right)$ and $g=\operatorname{Gal}\left(k^{\prime} / k\right)$, so that $G / H=g$. The group of $k^{\prime}$-rational points of the Jacobian variety of $X^{\prime}$ will be denoted by $J_{K}$. Since $X^{\prime}$ has a $k^{\prime}$-rational point, we have $J_{K}=\operatorname{Pic}^{0}\left(X^{\prime}\right)$. Similarly, let $J_{F}=\operatorname{Pic}^{0}(X)$. Then there exists a natural capitulation map $j_{K / F}: J_{F} \rightarrow J_{K}^{G}$, which is defined by pulling back line bundles. Its kernel was determined in [22, Theorem 5] under the assumption that $k=k^{\prime}$. In general, the following holds

Theorem 8.1. Let $K / F$ be a finite Galois extension of function fields and let $M$ be the maximal abelian unramified extension of $k^{\prime} F$ in $K$. Then the kernel of the capitulation map $j_{K / F}: J_{F} \rightarrow J_{K}^{G}$ is naturally isomorphic to $\operatorname{Hom}_{g}\left(\operatorname{Gal}\left(M / F k^{\prime}\right),\left(k^{\prime}\right)^{*}\right)$.

Proof. The Hochschild-Serre spectral sequence

$$
H^{p}\left(g, H^{q}\left(H,\left(k^{\prime}\right)^{*}\right)\right) \Longrightarrow H^{p+q}\left(G,\left(k^{\prime}\right)^{*}\right)
$$

yields an exact sequence

$$
0 \rightarrow H^{1}\left(g,\left(k^{\prime}\right)^{*}\right) \rightarrow H^{1}\left(G,\left(k^{\prime}\right)^{*}\right) \rightarrow H^{1}\left(H,\left(k^{\prime}\right)^{*}\right)^{g} \rightarrow \operatorname{Br}\left(k^{\prime} / k\right)
$$

(see, for example, [21, p.309]). Now $H^{1}\left(g,\left(k^{\prime}\right)^{*}\right)=0$ by Hilbert's Theorem 90 and $\operatorname{Br}\left(k^{\prime} / k\right)=0$ since the Brauer group of a finite field is zero (see, e.g., [24]). Therefore

$$
\begin{aligned}
H^{1}\left(G,\left(k^{\prime}\right)^{*}\right) & \simeq H^{1}\left(H,\left(k^{\prime}\right)^{*}\right)^{g} \simeq \operatorname{Hom}_{g}\left(H,\left(k^{\prime}\right)^{*}\right) \\
& \simeq \operatorname{Hom}_{g}\left(\operatorname{Gal}\left(M^{\prime} / F k^{\prime}\right),\left(k^{\prime}\right)^{*}\right)
\end{aligned}
$$

where $M^{\prime}$ is the maximal abelian extension of $F k^{\prime}$ in $K$. Taking into account these facts, it is not difficult to adapt the proof of Theorem 5 of [22] to the case where $k^{\prime}$ is not necessarily equal to $k$. We leave the details to the reader.

We will now study the cokernel of the capitulation map $J_{F} \rightarrow J_{K}^{G}$. Let $\mathcal{I}_{K}^{0}=$ $\operatorname{Div}^{0}\left(X^{\prime}\right)$. Then there exists a natural exact sequence

$$
1 \rightarrow\left(k^{\prime}\right)^{*} \rightarrow K^{*} \rightarrow \mathcal{I}_{K}^{0} \rightarrow J_{K} \rightarrow 0
$$

inducing an exact sequence

$$
1 \rightarrow\left(K^{*} /\left(k^{\prime}\right)^{*}\right)^{G} \rightarrow\left(\mathcal{I}_{K}^{0}\right)^{G} \rightarrow J_{K}^{G} \rightarrow H^{1}\left(G, K^{*} /\left(k^{\prime}\right)^{*}\right) \rightarrow H^{1}\left(G, \mathcal{I}_{K}^{0}\right)
$$

Define

$$
\left(J_{K}^{G}\right)_{\text {trans }}=\operatorname{Ker}\left[J_{K}^{G} \rightarrow H^{1}\left(G, K^{*} /\left(k^{\prime}\right)^{*}\right)\right] .
$$

Then the image of $j_{K / F}$ is contained in $\left(J_{K}^{G}\right)_{\text {trans }}$. Let $j_{K / F}^{\prime}: J_{F} \rightarrow\left(J_{K}^{G}\right)_{\text {trans }}$ be the map induced by $j_{K / F}$. Arguing as in $\S 2$ (see, especially, diagram (8)), we obtain the following result. 
Proposition 8.2. There exists a natural exact sequence

$$
0 \rightarrow \operatorname{Ker} j_{K / F} \rightarrow H^{1}\left(G,\left(k^{\prime}\right)^{*}\right) \rightarrow\left(\mathcal{I}_{K}^{0}\right)^{G} / \mathcal{I}_{F}^{0} \rightarrow \text { Coker } j_{K / F}^{\prime} \rightarrow 0 .
$$

Now define positive integers $\delta$ and $\delta^{\prime 10}$ by

$$
\begin{aligned}
& \operatorname{Im}\left[\operatorname{Div}\left(X^{\prime}\right)^{G} \stackrel{\operatorname{deg}}{\longrightarrow} \mathbb{Z}\right]=\delta \mathbb{Z} \\
& \operatorname{Im}\left[\operatorname{Pic}\left(X^{\prime}\right)^{G} \stackrel{\operatorname{deg}}{\longrightarrow} \mathbb{Z}\right]=\delta^{\prime} \mathbb{Z} .
\end{aligned}
$$

The integer $\delta$ is "fairly easy to compute" [22, p.164]. Indeed, it can be shown that, if $P_{1}, P_{2}, \ldots, P_{r}$ are the primes of $F$ that ramify in $K$ and $e_{1}, e_{2}, \ldots, e_{r}$ are their respective ramification indices, then

$$
\delta=\left(n,\left(n / e_{1}\right) \operatorname{deg}_{F} P_{1}, \ldots,\left(n / e_{r}\right) \operatorname{deg}_{F} P_{r}\right) .
$$

See [22, proof of Proposition 1, p.163] (by contrast, $\delta^{\prime}$ is a "more subtle invariant" [op.cit., p.164]). Clearly, $\delta^{\prime}|\delta| n$.

Lemma 8.3. There exists a natural exact sequence

$$
0 \rightarrow\left(\mathcal{I}_{K}^{0}\right)^{G} / \mathcal{I}_{F}^{0} \rightarrow \bigoplus_{i=1}^{r} \mathbb{Z} / e_{i} \mathbb{Z} \rightarrow \delta \mathbb{Z} / n \mathbb{Z} \rightarrow 0
$$

Proof. This follows at once from [22, Proposition 1 and Theorem 3(B)].

We also have the following exact sequence, which is the analog of Proposition 2.2 in this context.

Proposition 8.4. There exists an exact sequence

$$
0 \rightarrow \operatorname{Coker} j_{K / F}^{\prime} \rightarrow \text { Coker } j_{K / F} \rightarrow H^{1}\left(G, K^{*} /\left(k^{\prime}\right)^{*}\right) \rightarrow \Gamma \rightarrow 0,
$$

where $\Gamma$ is a cyclic group of order $\delta / \delta^{\prime}$.

Proof. This is immediate from (24) and [22, Theorem 3(A), p.163], which shows that the image, $\Gamma$, of $H^{1}\left(G, K^{*} /\left(k^{\prime}\right)^{*}\right)$ in $H^{1}\left(G, \mathcal{I}_{K}^{0}\right)$ under the map appearing in (24) is a cyclic group of the indicated order.

Combining statements 8.2, 8.3 and 8.4, we obtain the following analogue of C.Chevalley's classical "ambiguous class number formula" (cf. [22, Theorem 8, p.166]):

Theorem 8.5. We have

$$
\frac{\left[J_{K}^{G}\right]}{\left[J_{F}\right]}=\frac{\left[H^{1}\left(G, K^{*} /\left(k^{\prime}\right)^{*}\right)\right] \prod_{i=1}^{r} e_{i}}{\left(n / \delta^{\prime}\right)\left[H^{1}\left(G,\left(k^{\prime}\right)^{*}\right)\right]} .
$$

\footnotetext{
${ }^{10}$ One might call these integers the "index" and the "period" of $K / F$, respectively.
} 
Now we note that $(23)$ induces an exact sequence

$$
0 \rightarrow H^{1}\left(G, K^{*} /\left(k^{\prime}\right)^{*}\right) \rightarrow H^{2}\left(G,\left(k^{\prime}\right)^{*}\right) \rightarrow \operatorname{Br}(K / F) \rightarrow H^{2}\left(G, K^{*} /\left(k^{\prime}\right)^{*}\right)
$$

(cf. (4)). On the other hand, there exists a natural map $\operatorname{Br}(K / F) \rightarrow H^{2}\left(G, \mathcal{I}_{K}\right)$ (where $\mathcal{I}_{K}=\operatorname{Div}\left(X^{\prime}\right)$ ) which factors as

$$
\operatorname{Br}(K / F) \rightarrow H^{2}\left(G, K^{*} /\left(k^{\prime}\right)^{*}\right) \rightarrow H^{2}\left(G, \mathcal{I}_{K}\right),
$$

where the second map is induced by the canonical map $K^{*} /\left(k^{\prime}\right)^{*} \rightarrow \mathcal{I}_{K}$. We conclude that there exists an exact sequence

$$
0 \rightarrow H^{1}\left(G, K^{*} /\left(k^{\prime}\right)^{*}\right) \rightarrow H^{2}\left(G,\left(k^{\prime}\right)^{*}\right) \rightarrow B
$$

where

$$
\mathrm{B}=\operatorname{Ker}\left[\operatorname{Br}(K / F) \rightarrow H^{2}\left(G, \mathcal{I}_{K}\right)\right]
$$

Now essentially the same argument which proves Lemma 3.2 yields an isomorphism

$$
B \simeq \operatorname{Ker}\left[\bigoplus_{i=1}^{r} \mathbb{Z} / d_{i} \mathbb{Z} \stackrel{\Sigma}{\longrightarrow} \mathbb{Z} /\left[d_{1}, d_{2}, \ldots, d_{r}\right] \mathbb{Z}\right],
$$

where $d_{i}=\left[H^{2}\left(G_{P_{i}}, U_{P_{i}}\right)\right]$ for each $i=1,2, \ldots, r$ and $\left[d_{1}, d_{2}, \ldots, d_{r}\right]$ denotes the least common multiple of $d_{1}, d_{2}, \ldots, d_{r}$. In particular, $B$ is a group of order $\left(\prod_{i=1}^{r} d_{i}\right) /\left[d_{1}, d_{2}, \ldots, d_{r}\right]$. Consequently, if $K / F$ is cyclic, we have

$$
[B]=\frac{\prod_{i=1}^{r} e_{i}}{\left[e_{1}, e_{2}, \ldots, e_{r}\right]} .
$$

Now define

$$
m\left(P_{i}\right)=\left(\frac{q^{\operatorname{deg}_{F} P_{i}}-1}{\left(q^{\operatorname{deg}_{F} P_{i}}-1, e_{i}\right)}, q-1\right) \quad(1 \leq i \leq r)
$$

and set

$$
m=\left(m\left(P_{1}\right), m\left(P_{2}\right), \ldots, m\left(P_{r}\right)\right) .
$$

The following proposition shows that the degrees and the ramification indices of the primes that ramify in a cyclic extension $K / F$ are subject to certain non-obvious constraints.

Proposition 8.6. Assume that $K / F$ is a cyclic extension. Then

$$
\frac{\prod_{i=1}^{r} e_{i}}{\left[e_{1}, e_{2}, \ldots, e_{r}\right]} \equiv 0\left(\bmod \frac{q-1}{m}\right)
$$

where $m$ is the integer (27). Consequently, if the ramification indices $e_{i}$ are pairwise coprime, then

$$
\frac{q^{\operatorname{deg}_{F} P_{i}}-1}{\left(q^{\operatorname{deg}_{F} P_{i}}-1, e_{i}\right)} \equiv 0 \quad(\bmod q-1)
$$

for every $i=1,2, \ldots, r$.

Proof. The periodicity of the cohomology of cyclic groups and [22, Theorem 2(B), p.161, and Proposition 2, p.166] show that

$$
\frac{\left[H^{2}\left(G,\left(k^{\prime}\right)^{*}\right)\right]}{\left[H^{1}\left(G, K^{*} /\left(k^{\prime}\right)^{*}\right)\right]}=\frac{q-1}{m} .
$$

Therefore, $(q-1) / m$ divides $[B]=\left(\prod_{i=1}^{r} e_{i}\right) /\left[e_{1}, e_{2}, \ldots, e_{r}\right]$, as claimed. Now, if the ramification indices $e_{i}$ are pairwise coprime, then necessarily $m=q-1$. The definition of $m$ now yields the second assertion of the proposition. 
We conclude this paper with the following strengthening of [22, Theorem 14].

Theorem 8.7. Assume that $K / F$ is a Galois extension with Galois group $G \simeq$ $\bigoplus_{i=1}^{s} \mathbb{Z} / \ell \mathbb{Z}$, where $s$ is a positive integer and $\ell$ is a prime which divides $q-1$. Assume, furthermore, that the field of constants of $F$ is algebraically closed in $K$. Then

$$
\operatorname{rank}_{\ell}\left(J_{K}^{G}\right) \geq(s(s+1) / 2)-r .
$$

Proof. By (26), $\operatorname{rank}_{\ell}(B) \leq r-1$. Consequently, (25) shows that

$$
\operatorname{rank}_{\ell}\left(H^{2}\left(G,\left(k^{\prime}\right)^{*}\right)\right)=\operatorname{rank}_{\ell}\left(H^{2}\left(G, k^{*}\right)\right) \leq \operatorname{rank}_{\ell}\left(H^{1}\left(G, K^{*} / k^{*}\right)\right)+r-1 .
$$

The rest of the proof is similar to that of Theorem 14 of [22].

\section{APPENDIX}

The integers $\left[H^{2}\left(G_{w}, U_{w}\right)\right]$, where $w$ lies above a ramified prime of $K / F$, intervene at various places in the paper. The following result (which may be well-known) relates these integers to the ramification indices $e_{v}$ of $K / F$.

Proposition A.1. Let $v$ be a non-archimedean prime of $F$ and let $w$ be a fixed prime of $K$ lying above $v$. Then there exists an exact sequence

$$
0 \rightarrow \mathbb{Z} /\left(e_{v}, f_{v}\right) \mathbb{Z} \rightarrow H^{2}\left(G_{w}, U_{w}\right) \rightarrow \mathbb{Z} / e_{v} \mathbb{Z},
$$

where $f_{v}$ is the residue class degree. In particular, $\left[H^{2}\left(G_{w}, U_{w}\right)\right]$ divides $e_{v}^{2}$.

Proof. Let $F_{v}^{\text {un }}$ be the maximal unramified extension of $F_{v}$ contained in $K_{w}$. Set $I_{w}=\operatorname{Gal}\left(K_{w} / F_{v}^{\text {un }}\right)$. Then $I_{w}$ is a subgroup of $G_{w}$ of order $e_{v}$ and $G_{w} / I_{w}=$ $\operatorname{Gal}\left(F_{v}^{\mathrm{un}} / F_{v}\right)$ is a cyclic group of order $f_{v}$. The exact sequence of terms of low degree belonging to the Hochschild-Serre spectral sequence $H^{p}\left(G_{w} / I_{w}, H^{q}\left(I_{w}, U_{w}\right)\right) \Longrightarrow$ $H^{p+q}\left(G_{w}, U_{w}\right)$ yields, since $H^{i}\left(G_{w} / I_{w}, U_{w}^{I_{w}}\right)=0$ for all $i \geq 1$, an exact sequence

$$
0 \rightarrow H^{1}\left(G_{w} / I_{w}, H^{1}\left(I_{w}, U_{w}\right)\right) \rightarrow H^{2}\left(G_{w}, U_{w}\right) \rightarrow H^{2}\left(I_{w}, U_{w}\right)
$$

(see [21, p.309]). On the other hand, since $K_{w} / F_{v}^{\text {un }}$ is totally ramified, we have $H^{2}\left(I_{w}, U_{w}\right) \simeq H^{2}\left(I_{w}, K_{w}^{*}\right) \simeq \mathbb{Z} / e_{v} \mathbb{Z}$, by [24, Ch.XII, Exer.2(b), p.182] and local class field theory. Further, $H^{1}\left(I_{w}, U_{w}\right)=\mathbb{Z} / e_{v} \mathbb{Z}$ with trivial $G_{w} / I_{w}$-action [op.cit.], whence

$$
H^{1}\left(G_{w} / I_{w}, H^{1}\left(I_{w}, U_{w}\right)\right) \simeq \operatorname{Hom}\left(\mathbb{Z} / f_{v} \mathbb{Z}, \mathbb{Z} / e_{v} \mathbb{Z}\right) \simeq \mathbb{Z} /\left(e_{v}, f_{v}\right) \mathbb{Z} .
$$

We conclude that there exists an exact sequence

$$
0 \rightarrow \mathbb{Z} /\left(e_{v}, f_{v}\right) \mathbb{Z} \rightarrow H^{2}\left(G_{w}, U_{w}\right) \rightarrow \mathbb{Z} / e_{v} \mathbb{Z},
$$

as asserted.

Now define integers $d_{v}^{\prime}$ by

$$
d_{v}^{\prime}=\left\{\begin{array}{cl}
{\left[K_{w}: F_{v}\right]} & \text { if } v \in S \\
e_{v} & \text { if } v \in R \backslash S .
\end{array}\right.
$$

The following corollary shows that the hypothesis of Corollary 3.5 is satisfied if the integers $d_{v}^{\prime}$ defined above (which are relatively easy to compute) are pairwise coprime.

Corollary A.2. Assume that the integers $d_{v}^{\prime}$, where $v \in S^{\prime}$, are pairwise coprime. Then so also are the integers $d_{v}$ defined by (18). 


\section{REFERENCES}

1. Artin, E. and Tate, J., Class Field Theory, Benjamin, New York-Amsterdam, 1967.

2. Atiyah, M. and Wall, C., Cohomology of groups, in: Algebraic Number Theory, Cassels, J.W.S. and Fröhlich, A. (Eds.), Academic Press, London, 1967, pp. 94-115.

3. Auslander, M. and Brumer, A., Galois cohomology and the Brauer group of commutative rings, (unpublished).

4. Bond, R., Some results on the Capitulation Problem, J. Number Theory 13 (1981), 246-254.

5. Brumer, A., Galois groups of extensions of algebraic number fields with given ramification, Michigan Math. J. 13 (1966), 33-40.

6. Chase, S., Harrison, D. and Rosenberg, A., Galois theory and cohomology of commutative rings, Mem. Amer. Math. Soc. (1965), no. 52.

7. Fleckinger, V. and Thiébaud, C., Idéaux ambiges dans les corps de fonctions, J. Number Theory 100 (2003), 217-228.

8. Furuya, H., Principal ideal theorems in the genus field for absolutely abelian extensions, J. Number Theory 9 (1977), 4-15.

9. González-Avilés, C., Finite modules over non-semisimple group rings, Israel J. Math. 144 (2004), 61-92.

10. (2003), no. 2, 391-419.

11. Gras, G., Class Field Theory. From Theory to Practice, Springer, New York, NY, 2003.

12. Gruenberg, K. and Weiss, A., Capitulation and transfer kernels, J. Théor. Nombres Bordeaux 12 (2003), no. 1, 219-226.

13. Ichimura, H., On a quotient of the unramified Iwasawa module over an abelian number field, J. Number Theory 88 (2001), 175-190.

14. A note on the ideal class group of the cyclotomic $\mathbb{Z}_{p}$-extension of a totally real number field, Acta Arith. 105 (2002), 29-34.

15. On a quotient of the unramified Iwasawa module over an abelian number field, II, Pacific J. Math. 206 (2002), 129-137.

16. Iwasawa, K., On cohomology groups of units for $\mathbb{Z}_{p}$-extensions, Amer.J. Math. 105 (1983), 189-200.

17. Kisilevsky, H., Some results related to Hilbert's Theorem 94, J. Number Theory 2 (1970), 199-206.

18. Le Floc'h, M., Movahhedi, A. and Nguyen Quang Do, T., On capitulation cokernels in Iwasawa Theory, Amer. J. Math. 127 (2005), no. 4, 851-877.

19. Maire, C., Extensions T-ramifiées modérées, S-décomposées, Dissertation, Université de Franche-Comté, 1995.

20. Maire, C., T-S capitulation, Publ. Math. Fac. Sci. Besançon, Univ. Franche-Comté, Besançon, (1997).

21. Milne, J.S., Étale Cohomology, Princeton University Press, Princeton, N.J., 1980.

22. Rosen, M., Ambiguous divisor classes in function fields, J. Number Theory 9 (1977), 160-174.

23. Schmithals, B., Kapitulation der idealklassen und einheitenstruktur in zahlkörpern, J. Reine Angew. Math. 358 (1985), 43-60.

24. Serre, J.-P., Local Fields, Grad.Texts in Math., vol. 67, Springer-Verlag, New York, 1979.

25. Suzuki, H., A generalization of Hilbert's Theorem 94, Nagoya Math.J. 121 (1991), 161-169.

26. Tate, J., Global Class Field Theory, in: Algebraic Number Theory, Cassels, J.W.S. and Fröhlich, A. (Eds.), Academic Press, London, 1967, pp. 162-203.

27. Thiébaud, C., Sur la capitulation dans les corps de genres d'une extension abélienne d'un corps quadratique imaginaire, J. Number Theory 85 (2000), 92-107.

28. van der Waall, H., Galois cohomology and class groups, Master's degree thesis, Universiteit van Amsterdam, 1994. http://www.cecm.sfu.ca/ awaall.

29. Yokoi, H., On the class number of a relatively cyclic number field, Nagoya Math. J. 29 (1967), 31-44.

Departamento de Matemáticas, Universidad Andrés Bello, República 252, SantiAgo, Chile.

E-mail address: cristiangonzalez@unab.cl 